\title{
Diseño de mezclas asfálticas integrando residuos sólidos de la industria automovilística (elastómero) y de vías (pavimento asfáltico envejecido) en Manabí, Ecuador
}

Gabriel ANTONio NaVARRETe SChETtini ${ }^{1}$

RECIBIDO: 11/05/2018 ACEPTADO: 05/12/2018

\begin{abstract}
RESUMEN
El propósito de la investigación es diseñar mezclas asfálticas integrando residuos sólidos de neumáticos fuera de uso (elastómero) y pavimento asfáltico envejecido en el Cantón Bolívar, provincia de Manabí, Ecuador. Los factores en estudio fueron el elastómero en los niveles $4 \%, 8 \%, 12 \%$ y $16 \%$, y la granulometría fina, media y gruesa, empleando $52 \%$ de pavimento asfáltico envejecido. Además, se empleó el diseño completamente al azar de un arreglo bifactorial GxE+1 con trece tratamientos y tres repeticiones, donde $G$ corresponde a la granulometría de áridos utilizados y $E$ al elastómero. A partir del buen proceder del diseño de mezclas asfálticas con pavimento asfáltico envejecido, podemos concluir que, de la adición de 4\%,8\% y $12 \%$ de elastómero en las curvas granulométricas media y fina, es posible utilizar aquel diseño en vías de tráfico liviano y medio, como el que se presenta en el Cantón Bolívar.
\end{abstract}

Palabras-claves: Elastómero; pavimento asfáltico envejecido; residuos de industrias.

\section{INTRODUCCIÓN}

En el mundo hay aproximadamente 800 millones de vehículos comerciales en uso y cerca de 70 millones de unidades se añaden a ese número cada año (Bureau of International Recycling, 2016). Además de eso, de acuerdo con Peláez, Velásquez y Giraldo (2017), en 2014 se estimó que el consumo mundial de esta materia prima fue de 28,9 millones de toneladas, aumentando en un $0,7 \%$ en el 2015. De toda esa cantidad mundial mencionada, en "Ecuador cada año se desechan alrededor de 2.4 millones de neumáticos" (Castro, 2015, p. 2) y, al ser ideado como un producto que ofrece la mayor resistencia posible al desgaste en su uso, se estima que su descomposición natural ocurrirá en un periodo mayor a 600 años. La composición de estos neumáticos incluye elementos peligrosos como el plomo, el cromo, el cadmio y otros metales pesados que, cuando se eliminan de manera inapropiada, constituyen una amenaza para la salud y el medio ambiente (Bertalot, 2017). El reciclado de pavimentos asfálticos se realiza sobre materiales deteriorados que han perdido en gran medida sus propiedades iniciales. El aprovechamiento de los materiales fresados mediante el reciclado resulta técnicamente viable, lo cual se enriquece con la adición de materiales pétreos (Centro de Estudios y Experimentación de Obras Públicas, 2011).

Por otro lado, Sánchez (2012) señala que "los materiales que se obtienen tras el tratamiento de los residuos de neumáticos, una vez separados los restos aprovechables en la industria, pueden [tener varios usos]" (p. 29). Además, los estudiosos Sánchez (2012), Martín (2015) y Grytsenko, Pozdniakova y Vnukova (2015) coinciden en mencionar que una de las aplicaciones del caucho es funcionar como componente de las capas asfálticas que se usan en la construcción de carreteras, con lo que disminuye la extracción de áridos en canteras. Asimismo, le confieren características especiales, ya que puede ser utilizado como caucho en la capa de rodadura, para que ayude a obtener una mayor media de vida, más elasticidad, menos deformaciones, mayor resistencia al agrietamiento, mayor resistencia al arrastramiento. Aparte de lo dicho, también tiene la característica de

1 Ingeniero civil por la Universidad Central del Ecuador y magíster en Educación y Desarrollo Social de la Universidad Tecnológica Equinoccial. Actualmente, es docente de la Escuela Superior Politécnica Agropecuaria de Manabí Manuel Félix López (ESPAM MFL). Manabí, Ecuador

E-mail: gnavarrete@espam.edu.ec 
pavimento drenante y le otorga a la mezcla asfáltica impedimento en la acumulación de agua, lo que incrementa su adherencia, evita las proyecciones de agua y concede buenas condiciones ópticas y bajo nivel de ruido. De esta forma, presenta tanto beneficios ambientales como económicos, ya que las vías, con la incorporación de caucho, se vuelven más resistentes, durables y menos frágiles, incluso ofrecen oposición a que se quiebren y tiene una vida útil de 10 años (Amorim y Lima, 2018).

Por otro lado, Lacamara (2014) nos ofrece un dato relevante, el cual evidencia que "se pueden emplear entre 1000 y 7000 neumáticos por kilómetro de carretera de dos carriles. Cifras tan elevadas colocan el pavimento asfáltico como una de las grandes soluciones para emplear los neumáticos fuera de uso" (párr. 13). Así, el reciclado de pavimentos constituye una alternativa de rehabilitación de pavimentos más competitiva y sostenible, puesto que permite minimizar la utilización de recursos no renovables, agregados naturales y asfalto; además, previene la creación de residuos y la ocupación de botaderos (Paiva y Ramos, 2014). Asimismo, comprende una serie de técnicas constructivas con tendencia al aprovechamiento integral de materiales envejecidos de firmes y pavimentos. Por ello, es de vital importancia desarrollar una propuesta sostenible para las personas que

[...] basan sus ingresos en la recuperación informal de lo que otros desechan, están expuestos a peligros [sanitarios] [...]. Dentro de este sector informal, es importante el número de niños y niñas que encuentran en el trabajo de recuperación de residuos la única forma de sobrevivir ante una sociedad muchas veces indiferente (Lecitra, 2010, p. 4)

De esta forma, se propone diseñar mezclas asfálticas integrando residuos sólidos de neumáticos fuera de uso (elastómero) y pavimento asfáltico envejecido en el Cantón Bolívar, provincia de Manabí, Ecuador.

\section{METODOLOGÍA}

1. Diseño experimental. En la investigación se utilizó el diseño completamente al azar (DCA) en un arreglo bifactorial $\mathrm{GxE}+1$ con trece tratamientos y tres repeticiones, donde $\mathrm{G}$ corresponde a la granulometría y $\mathrm{E}$, al elastómero. Se utilizó el software InfoStad para determinar el coeficiente de variación (CV\%), y en las variables donde se constató diferencias estadísticas se realizó la prueba de Tukey al $5 \%$ con probabilidad del error.

2. Dosificación de los áridos. La dosificación de los materiales y la tolerancia fueron determinadas de acuerdo con los valores de la Tabla 1, la cual es la recomendada por las especificaciones generales para la construcción de caminos y puentes realizados (Ministerio de Transportes y Obras Públicas del Ecuador, 2002).

3. Granulometría de los materiales. Se realizó el ensayo de abrasión al material de $1 / 2$, el cual se realizó con la gradación $B$, que consiste en obtener material extraído de la granulometría, utilizando $2500 \mathrm{~g} \pm 2$ pasante del tamiz $19 \mathrm{~mm}$ o N. ${ }^{\circ} 3 / 4$, retenidos en el $12,5 \mathrm{~mm}$ o $\mathrm{N}^{\circ} 1 / 2$ y $2500 \mathrm{~g} \pm 2$, pasante del tamiz $12,5 \mathrm{~mm}$ o $\mathrm{N} .^{\circ} 1 / 2$, retenidos en el $9,5 \mathrm{~mm}$

Tabla 1. Especificaciones generales para la construcción de caminos y puentes.

\begin{tabular}{|c|c|c|c|c|}
\hline \multirow[t]{2}{*}{ Tamiz } & \multicolumn{4}{|c|}{ Porcentaje en peso que pasa a través de los tamices de malla cuadrada } \\
\hline & N. $.^{\circ} 3 / 4$ & N. ${ }^{\circ} 1 / 2$ & N. ${ }^{\circ} 3 / 8$ & N. ${ }^{\circ} 4$ \\
\hline N. ${ }^{\circ} 1(25,4 \mathrm{~mm})$ & 100 & & & \\
\hline $\mathrm{N} .^{\circ} 3 / 4(19,0 \mathrm{~mm})$ & $90-100$ & 100 & & \\
\hline N. ${ }^{\circ} 1 / 2(12,7 \mathrm{~mm})$ & & $90-100$ & 100 & \\
\hline $\mathrm{N} .^{\circ} 3 / 8(9,50 \mathrm{~mm})$ & $56-80$ & & $90-100$ & 100 \\
\hline N. ${ }^{\circ} 4(4,75 \mathrm{~mm})$ & $35-65$ & $44-74$ & $55-85$ & $80-100$ \\
\hline N. ${ }^{\circ} 8(2,36 \mathrm{~mm})$ & $23-49$ & $28-58$ & $32-67$ & $65-100$ \\
\hline N. ${ }^{\circ} 16(1,18 \mathrm{~mm})$ & & & & $40-80$ \\
\hline $\mathrm{N} .^{\circ} 30(0,60 \mathrm{~mm})$ & & & & $25-65$ \\
\hline $\mathrm{N} .^{\circ} 50(0,30 \mathrm{~mm})$ & $5-19$ & $5-21$ & $7-23$ & $7-40$ \\
\hline $\mathrm{N} .^{\circ} 100(0,15 \mathrm{~mm})$ & & & & $3-20$ \\
\hline $\mathrm{N} .{ }^{\circ} 200(0,075 \mathrm{~mm})$ & $2-8$ & $2-10$ & $2-10$ & $2-10$ \\
\hline
\end{tabular}

Fuente: Ministerio de Transporte y Obras Públicas del Ecuador (2002). 
o N. ${ }^{\circ} 3 / 8$, y se coloca en la máquina de los Ángeles, donde se dan 500 vueltas. Una vez terminado, se saca el material y se lo tamiza por el $1,70 \mathrm{~mm}$ o $\mathrm{N}$. $^{\circ}$ 12 , se lava y se seca a $11{ }^{\circ} \mathrm{C}$. Luego de secarlo, se procede a pesarlo para su cálculo de coeficiente de desgaste.

El ensayo de masa unitaria o peso volumétrico consistió en tener los materiales (1/2, 3/8, cisco y arena) totalmente secos, moldes metálicos con pesos y volúmenes conocidos para realizar el ensayo, el cual consiste en añadir material de una forma constante hasta llenar el recipiente enrazando y pesando. Así, se repite el procedimiento por 3 ocasiones para conocer la masa unitaria suelta. Por otro lado, para la masa unitaria compactada se llena el recipiente en tres capas iguales y se da 25 varilladas por capa. Una vez realizados los ensayos a los materiales de 1/2 (árido 1), 3/8 (árido 2), cisco (árido 3) y arena (árido 4) se procede a la digitación y cálculo de resultados, como se muestra en la Tabla 2.

4. Determinación del porcentaje óptimo de asfalto. Ello se obtiene con el porcentaje óptimo de asfalto definido a partir de la curva de vacíos, con la cual se obtuvo $6,2 \%$, como se observa en la Tabla
3. Además, se procedió a conseguir los otros resultados de las curvas, tales como pesos unitarios (densidad), vacíos, estabilidad, vacíos de agregado mineral, flujo y VFA.

5. Integración del pavimento asfáltico envejecido y del elastómero. El moldeo y ensayo de las briquetas en la investigación se las realizó de forma similar, solo con la inclusión del polvo de neumáti$\mathrm{co}$, pasante del tamiz $\mathrm{N} .^{\circ} 10$ y retenido en el tamiz $\mathrm{N}{ }^{\circ} 40$ y pavimento asfáltico envejecido. Los agregados pétreos fueron mezclados individualmente sin adición de residuos sólidos, manteniendo temperaturas con máximos y mínimos $\left(160^{\circ} \mathrm{C}-80^{\circ} \mathrm{C}\right)$. De acuerdo con Figueroa, Fonseca, Amaya y Prieto (2008), a esta temperatura se presenta un mejor proceso de mezclado, logrando una mayor homogenización en el ligante asfáltico modificado. En la Tabla 4 se muestran los valores promedio de las exigencias del diseño de Marshall, donde se utilizó $52 \%$ de pavimento asfáltico envejecido y se determinó que el pavimento asfáltico no cumple con las exigencias propuestas por el Ministerio de Transporte y Obras Públicas del Ecuador (2002) y Garni-

Tabla 2. Mezcla de los agregados.

\begin{tabular}{|c|c|c|c|c|c|c|c|}
\hline \multicolumn{8}{|c|}{ Granulometría combinada para la mezcla } \\
\hline \multirow{2}{*}{$\begin{array}{l}\text { Tipo de } \\
\text { material }\end{array}$} & \multirow{2}{*}{$\begin{array}{l}\text { Porcentajes } \\
\text { para mezcla }\end{array}$} & \multicolumn{6}{|c|}{ Porcentajes pasantes en tamices } \\
\hline & & N. ${ }^{\circ} 3 / 4$ & N. ${ }^{\circ} 1 / 2$ & N. ${ }^{\circ} 4$ & N..$^{\circ} 8$ & N. ${ }^{\circ} 50$ & N. ${ }^{\circ} 200$ \\
\hline Árido $N .^{\circ} 1$ & $12 \%$ & 12,0 & 6,2 & 0,3 & 0,3 & 0,2 & 0,2 \\
\hline Árido $\mathrm{N} .^{\circ} 2$ & $28 \%$ & 28,0 & 28,0 & 4,9 & 1,1 & 0,9 & 0,7 \\
\hline Árido $\mathrm{N} .^{\circ} 3$ & $45 \%$ & 45,0 & 45,0 & 42,5 & 27,5 & 12,4 & 5,7 \\
\hline Árido N. ${ }^{\circ} 4$ & $15 \%$ & 15,0 & 15,0 & 14,9 & 14,5 & 4,4 & 0,2 \\
\hline \multicolumn{2}{|c|}{ Granulometría obtenida } & 100,0 & 94,2 & 62,5 & 43,3 & 18,0 & 6,8 \\
\hline \multicolumn{2}{|c|}{ Especificación } & 100 & $90-100$ & $44-77$ & $28-58$ & $5-21$ & $2-10$ \\
\hline \multicolumn{2}{|c|}{ Granulometría esperada } & 100,0 & 95,0 & 60,5 & 43,0 & 13,0 & 6,0 \\
\hline
\end{tabular}

Fuente: Elaboración propia.

Tabla 3. Diseño de Marshall para obtener el porcentaje óptimo de asfalto.

\begin{tabular}{|l|c|c|c|}
\hline \multicolumn{1}{|c|}{ Propiedades } & \% asfalto-diseño & Valor obtenido & Exigencias \\
\hline Estabilidad & 6,20 & 2546 & $8-1800 \mathrm{lb}$ \\
\hline Flujo & 6,20 & 12,0 & $<2000$ \\
\hline Peso unitario & 6,20 & 2281 & $3,0-5,0 \%$ \\
\hline Vacíos en mezcla & 6,20 & 4,0 & $>13 \%$ \\
\hline Vacíos en agregados & 6,20 & 15,20 & $65-75 \%$ \\
\hline Vacíos rellenos asfalto & 6,20 & 71 & \\
\hline
\end{tabular}

Fuente: Elaboración propia. 
ca, Delgado, Gómez, Alonso y Alarcón (2004), por lo que se incluyeron agregados pétreos.

Las Tablas 5, 6 y 7 muestran las granulometrías con $52 \%$ de pavimento asfáltico envejecido enriquecido con agregados pétreos.

Una vez establecido el porcentaje óptimo de asfalto en la mezcla, que resultó ser el $6,2 \%$, se procede a agregar, mediante el proceso de vía seca, el polvo de neumático en diferentes porcentajes, partiendo desde el $4 \%$ hasta el $16 \%$.

6. Variables a evaluar en las mezclas asfálticas. Se utilizó el diseño de Marshall para evaluar las mezclas asfálticas, se determinó la densidad bulk, la estabilidad, el flujo, los vacíos en la mezcla, los vacíos en agregados minerales y los vacíos en rellenos de

Tabla 4. Diseño de Marshall del pavimento asfáltico envejecido.

\begin{tabular}{|l|c|c|c|}
\hline \multicolumn{1}{|c|}{ Propiedades } & \% asfalto-diseño & Valor obtenido & Exigencias \\
\hline Estabilidad & 5,70 & 3381 & $>1800 \mathrm{lb}$ \\
\hline Flujo & 5,70 & 15,5 & $8-16 \mathrm{in} / 100$ \\
\hline Peso unitario & 5,70 & 2174 & $>2000$ \\
\hline Vacíos en mezcla & 5,70 & 6,37 & $3,0-5,0 \%$ \\
\hline Vacíos en agregados & 5,70 & 19,62 & $>13 \%$ \\
\hline Vacíos rellenos asfalto & 5,70 & 67,37 & $65-75 \%$ \\
\hline
\end{tabular}

Fuente: Elaboración propia.

Tabla 5. Graduación granulométrica centrada del pavimento asfáltico envejecido enriquecido con agregados pétreos.

\begin{tabular}{|c|c|c|c|c|c|c|c|}
\hline \multicolumn{8}{|c|}{ Graduación combinada para la mezcla } \\
\hline \multirow{2}{*}{$\begin{array}{l}\text { Tipo de } \\
\text { material }\end{array}$} & \multirow{2}{*}{$\begin{array}{l}\text { Porcentajes } \\
\text { para mezcla }\end{array}$} & \multicolumn{6}{|c|}{ Porcentajes pasantes en tamices } \\
\hline & & $N .^{\circ} 3 / 4$ & $N .^{\circ} 1 / 2$ & N. ${ }^{\circ} 4$ & N. ${ }^{\circ} 8$ & $N .^{\circ} 50$ & N. ${ }^{\circ} 200$ \\
\hline Árido $\mathrm{N} .^{\circ} 1$ & $10 \%$ & 10,0 & 4,8 & 0,2 & 0,2 & 0,1 & 0,1 \\
\hline Árido $\mathrm{N}^{\circ} 2$ & $16 \%$ & 16,0 & 16,0 & 2,3 & 0,7 & 0,5 & 0,4 \\
\hline Árido $\mathrm{N} .^{\circ} 3$ & $16 \%$ & 16,0 & 16,0 & 15,3 & 10,2 & 4,8 & 2,0 \\
\hline Árido $N .^{\circ} 4$ & $6 \%$ & 6,0 & 6,0 & 5,9 & 5,8 & 1,7 & 0,1 \\
\hline Reciclado & $52 \%$ & 52,0 & 50,4 & 37,7 & 25,8 & 10,5 & 2,0 \\
\hline \multicolumn{2}{|c|}{ Granulometría obtenida } & 100,0 & 93,2 & 61,4 & 42,7 & 17,6 & 4,6 \\
\hline \multicolumn{2}{|c|}{ Especificación } & 100 & $90-100$ & 44-77 & $28-58$ & $5-21$ & $2-10$ \\
\hline \multicolumn{2}{|c|}{ Granulometría esperada } & 100,0 & 95,0 & 60,5 & 43,0 & 13,0 & 6,0 \\
\hline
\end{tabular}

Fuente: Elaboración propia.

Tabla 6. Graduación granulométrica fina del pavimento asfáltico envejecido enriquecido con agregados pétreos.

\begin{tabular}{|c|c|c|c|c|c|c|c|}
\hline \multicolumn{8}{|c|}{ Graduación combinada para la mezcla } \\
\hline \multirow{2}{*}{$\begin{array}{l}\text { Tipo de } \\
\text { material }\end{array}$} & \multirow{2}{*}{$\begin{array}{l}\text { Porcentajes } \\
\text { para mezcla }\end{array}$} & \multicolumn{6}{|c|}{ Porcentajes pasantes en tamices } \\
\hline & & N. ${ }^{\circ} 3 / 4$ & N. ${ }^{\circ} 1 / 2$ & N..$^{\circ} 4$ & N. ${ }^{\circ} 8$ & N. ${ }^{\circ} 50$ & N. ${ }^{\circ} 200$ \\
\hline Árido $N .^{\circ} 1$ & $8 \%$ & 8,0 & 3,8 & 0,1 & 0,1 & 0,1 & 0,1 \\
\hline Árido $N .^{\circ} 2$ & $8 \%$ & 8,0 & 8,0 & 1,2 & 0,3 & 0,3 & 0,2 \\
\hline Árido $\mathrm{N}^{\circ} 3$ & $26 \%$ & 26,0 & 26,0 & 24,9 & 16,6 & 7,8 & 3,3 \\
\hline Árido $\mathrm{N} .^{\circ} 4$ & $6 \%$ & 6,0 & 6,0 & 5,9 & 5,8 & 1,7 & 0,1 \\
\hline Reciclado & $52 \%$ & 52,0 & 50,4 & 37,7 & 25,8 & 10,5 & 2,0 \\
\hline \multicolumn{2}{|c|}{ Granulometría obtenida } & 100,0 & 94,2 & 69,8 & 48,8 & 20,3 & 5,6 \\
\hline \multicolumn{2}{|c|}{ Especificación } & 100 & $90-100$ & 44-77 & $28-58$ & $5-21$ & $2-10$ \\
\hline \multicolumn{2}{|c|}{ Granulometría esperada } & 100,0 & 95,0 & 60,5 & 43,0 & 13,0 & 6,0 \\
\hline
\end{tabular}

Fuente: Elaboración propia. 
Tabla 7. Graduación granulométrica gruesa del pavimento asfáltico envejecido enriquecido con agregados pétreos.

\begin{tabular}{|c|c|c|c|c|c|c|c|}
\hline \multicolumn{8}{|c|}{ Graduación combinada para la mezcla } \\
\hline \multirow{2}{*}{$\begin{array}{l}\text { Tipo de } \\
\text { material }\end{array}$} & \multirow{2}{*}{$\begin{array}{l}\text { Porcentajes } \\
\text { para mezcla }\end{array}$} & \multicolumn{6}{|c|}{ Porcentajes pasantes en tamices } \\
\hline & & N. ${ }^{\circ} 3 / 4$ & N. ${ }^{\circ} 1 / 2$ & N. ${ }^{\circ} 4$ & N..$^{\circ} 8$ & N. ${ }^{\circ} 50$ & N. ${ }^{\circ} 200$ \\
\hline Árido $N .^{\circ} 1$ & $12 \%$ & 12,0 & 5,7 & 0,2 & 0,2 & 0,2 & 0,1 \\
\hline Árido $\mathrm{N} .^{\circ} 2$ & $22 \%$ & 20,0 & 22,0 & 3,2 & 0,9 & 0,7 & 0,5 \\
\hline Árido $\mathrm{N} .^{\circ} 3$ & $14 \%$ & 14,0 & 14,0 & 13,4 & 9,0 & 4,2 & 1,8 \\
\hline Árido N. ${ }^{\circ} 4$ & $2 \%$ & 2,0 & 2,0 & 2,0 & 1,9 & 0,6 & 0,0 \\
\hline Reciclado & $52 \%$ & 52,0 & 50,4 & 37,7 & 25,8 & 10,5 & 2,0 \\
\hline \multicolumn{2}{|c|}{ Granulometría obtenida } & 100,0 & 94,1 & 56,5 & 37,8 & 16,1 & 4,5 \\
\hline \multicolumn{2}{|c|}{ Especificación } & 100 & $90-100$ & $44-77$ & $28-58$ & $5-21$ & $2-10$ \\
\hline \multicolumn{2}{|c|}{ Granulometría esperada } & 100,0 & 95,0 & 60,5 & 43,0 & 13,0 & 6.0 \\
\hline
\end{tabular}

Fuente: Elaboración propia.

asfalto, de acuerdo al Manual de Especificaciones (Ministerio de Transporte y Obras Públicas del Ecuador, 2002 y Garnica et al., 2004).

\section{RESULTADOS Y DISCUSIÓN}

1. Densidad bulk. En la Tabla 8 se observan los valores promedios de la variable "Densidad bulk". El análisis de varianza (ADEVA) no determinó diferencias estadísticas para el factor $\mathrm{G}$, ni para el factor $E$, ni en la interacción GxE ni en la comparación factorial versus testigo.

2. Porcentaje de vacíos en la mezcla. En la Tabla 8 se observan los valores promedios de la variable "\% de vacíos en la mezcla"; el ADEVA no determinó diferencias estadísticas para el factor $\mathrm{E}$, ni en la interacción GxE, ni en la comparación factorial versus testigo, a excepción del factor $\mathrm{G}$. La prueba de significancia determinó dos rangos de similitud estadística en el factor $\mathrm{G}$, donde el mejor " $\%$ de vacíos en la mezcla" correspondió a G1 con 4,26\%, seguido de G2. En ese sentido, el Ministerio de Transporte y Obras Públicas del Ecuador (2002) señala que el $5 \%$ es el valor máximo exigido en el porcentaje de vacíos, por lo tanto la granulometría gruesa con el $5 \%$ no cumple con las exigencias; además, vale añadir que el porcentaje permitido de vacíos en muestras de laboratorio para capas de base y capas superficiales se encuentra entre $3 \%$ y $5 \%$.

3. Estabilidad. En la Tabla 8 se observan los valores promedios de la variable "Estabilidad", el ADEVA determinó diferencias estadísticas significativas para el factor $G$, el factor $E$, la interacción GxE y para la comparación factorial versus testigo. En la prueba de significancia realizada en el factor G, se establecieron dos rangos de similitud estadística, de los cuales fue $\mathrm{G} 2$ quien obtuvo mayor estabilidad con 2880,86 lb; en cambio, G3, con 2640,34 $\mathrm{lb}$, obtuvo la estabilidad más baja. Asimismo, en la prueba de significancia realizada al factor $E$, se determinaron tres rangos de similitud estadística, donde E3 se destacó con mayor estabilidad con $3018,59 \mathrm{lb}$; y E4, con $2472,87 \mathrm{lb}$, obtuvo la estabilidad más baja. En la prueba de significancia realizada a la interacción GxE, se establecieron cuatro rangos de similitud estadística, donde G2E3 (G media-12\% de elastómero) y G1E3 (G fina-12\% de elastómero) tuvieron $3119,73 \mathrm{lb}$ y $3042,36 \mathrm{lb}$, respectivamente. Además, G2E3 y G1E3 se destacaron con la mayor estabilidad, mientras que G3E4 (G gruesa-elastómero 16\%) obtuvo la estabilidad más baja con $2310,61 \mathrm{lb}$. En la comparación factorial versus testigo, se establecieron tres rangos de similitud estadística, donde destacaron el factor $\mathrm{G}$ con $2790,10 \mathrm{lb}$, seguido del factor $\mathrm{E}$ con 2640,10 lb y, en último lugar, el testigo con $2605,67 \mathrm{lb}$ de estabilidad.

Un pavimento estable es capaz de mantener su forma y lisura bajo cargas repetitivas [...] Las especificaciones de estabilidad deben ser lo suficiente altas para acomodar adecuadamente el tránsito esperado, pero no más altas de lo que exijan las condiciones de transito [sic] (Carrasco, 2004, p. 65).

Asimismo, el Ministerio de Transporte y Obras Públicas del Ecuador (2002) exige estabilidades mayores a $1800 \mathrm{lb}$, para que todos los tratamientos cumplan con las exigencias del diseño de Marshall.

4. Flujo. En la Tabla 9 se observan los valores promedios de la variable "Flujo"; el ADEVA no determinó diferencias estadísticas para la interacción 
Tabla 8. Densidad bulk, porcentaje de vacíos en la mezcla y estabilidad. Diseño de mezclas asfálticas integrando residuos sólidos de la industria automovilística (elastómero) y de vías (pavimento asfáltico envejecido).

\begin{tabular}{|c|c|c|c|}
\hline Tratamientos & Densidad bulk & $\%$ de vacíos en la mezcla & Estabilidad \\
\hline Factor G & NS & $* *$ & $* *$ \\
\hline G1 granulometría fina & 2282,17 & $4,26 \%(a)$ & $2849,09 \mathrm{lb}(\mathrm{a})$ \\
\hline G2 granulometría media & 2274,67 & $4,58 \%(a b)$ & $2880,86 \mathrm{lb}(\mathrm{a})$ \\
\hline G3 granulometría gruesa & 2272,50 & $5,00 \%(b)$ & $2640,34 \mathrm{lb}(\mathrm{b})$ \\
\hline Factor $\mathrm{E}$ & NS & NS & ** \\
\hline E1 4\% elastómero & 2270,11 & $4,73 \%$ & $2785,78 \mathrm{lb}(\mathrm{b})$ \\
\hline E2 8\% elastómero & 2274,89 & $4,60 \%$ & $2883,15 \mathrm{lb}(\mathrm{b})$ \\
\hline E3 $12 \%$ elastómero & 2276,56 & $4,46 \%$ & $3018,59 \mathrm{lb}(\mathrm{a})$ \\
\hline E4 $16 \%$ elastómero & 2284,22 & $4,64 \%$ & $2472,87 \mathrm{lb}(\mathrm{c})$ \\
\hline Interacción & NS & NS & ** \\
\hline G1E1 & 2282 & $4,15 \%$ & $2890,00 \mathrm{lb}(\mathrm{ab})$ \\
\hline G1E2 & 2284,67 & $4,27 \%$ & $2953,57 \mathrm{lb}(\mathrm{ab})$ \\
\hline G1E3 & 2279,33 & $4,49 \%$ & 2042,36 lb (a) \\
\hline G1E4 & 2282,67 & $4,13 \%$ & $2510,42 \mathrm{lb}(\mathrm{de})$ \\
\hline G2E1 & 2267,33 & $5,00 \%$ & $2870,94 \mathrm{lb}(\mathrm{abc})$ \\
\hline G2E2 & 2275,67 & $4,42 \%$ & $2935,18 \mathrm{lb}(\mathrm{ab})$ \\
\hline G2E3 & 2281,67 & $4,18 \%$ & $3119,73 \mathrm{lb}(\mathrm{a})$ \\
\hline G2E4 & 2274 & $4,72 \%$ & $2597,57 \mathrm{lb}(\mathrm{cd})$ \\
\hline G3E1 & 2261 & $5,05 \%$ & $2596,39 \mathrm{lb}(\mathrm{cd})$ \\
\hline G3E2 & 2264,33 & $5,12 \%$ & $2760,70 \mathrm{lb}(\mathrm{bcd})$ \\
\hline G3E3 & 2268,67 & $4,72 \%$ & $2893,68 \mathrm{lb}(\mathrm{ab})$ \\
\hline G3E4 & 2296 & $5,07 \%$ & $2310,61 \mathrm{lb}(\mathrm{e})$ \\
\hline Factorial versus testigo & NS & NS & ** \\
\hline Testigo absoluto & 2286,67 & $4,21 \%$ & $2605,67 \mathrm{lb}(\mathrm{c})$ \\
\hline Granulometría & 2278,11 & $4,61 \%$ & $2790,10 \mathrm{lb}(\mathrm{a})$ \\
\hline Elastómero & 2290,30 & $4,60 \%$ & $2640,10 \mathrm{lb}(\mathrm{b})$ \\
\hline Coeficiente de variación & 0,87 & 9,75 & 3,38 \\
\hline $\mathbf{P}$ & 0,6711 & 0,044 & $<0,0001$ \\
\hline
\end{tabular}

(*) Diferencia significativa entre los tratamientos.

$\left.{ }^{* *}\right)$ Diferencia altamente significativa entre los tratamientos. Letras en común no son estadísticamente diferentes.

Fuente: Elaboración propia.

GxE; por el contrario, se evidenciaron diferencias estadísticas para el factor $\mathrm{G}$, el factor $\mathrm{E}$ y la comparación factorial versus testigo. La prueba de significancia realizada al factor $\mathrm{G}$ estableció dos rangos de similitud estadística, donde resultaron $\mathrm{G} 2$ y $\mathrm{G} 1$ con $14,42 \mathrm{in} / 100$ y 14,83 in/100, respectivamente, y destacaron sobre la granulometría gruesa con 15,17 in/100. En la prueba estadística realizada al factor $E$, se establecieron dos rangos de similitud estadística, derivando E1, E2 y E3, con 14,44 in/100, 14,56 in/100 y $14,89 \mathrm{in} / 100$, respectivamente, los cuales destacaron con buenos valores de flujo en comparación a las 15,33 in/100 de E4. La prueba de significancia realizada a la comparación de la factorial versus el testigo estableció dos rangos de similitud estadística, de los cuales es el testigo quien obtuvo el mejor valor de flujo con $13 \mathrm{in} / 100$, seguido de los factores $G$ y $E$ con 14,81 in/100 en ambos casos.
De esta manera, las mezclas asfálticas con material reutilizable solo cumplen las exigencias para tráfico liviano y medio, lo que coincide con el Ministerio de Transporte y Obras Públicas del Ecuador (2002), que establece que el mínimo debe ser 8 in/100 para todos los tipos de tráfico, tales como, muy pesado, pesado, medio y liviano. El valor de flujo máximo exigido para los tráficos muy pesado y pesado será 14 in/100; en cambio, para los tráficos medio y liviano puede extenderse hasta el máximo de 16 in/100.

5. Vacíos en agregados minerales. En la Tabla 9 se observan los valores de la variable "\% de vacíos en agregados minerales"; el ADEVA no determinó diferencias estadísticas significativas para el factor E, interacción GxE y comparación factorial versus testigo; no obstante, determinó diferencias estadísticas para el factor $\mathrm{G}$. En la prueba de significancia realizada al factor $G$ se establecieron 
Tabla 9. Flujo, vacíos en agregados minerales y vacíos en rellenos de asfalto. Diseño de mezclas asfálticas integrando residuos sólidos de la industria automovilística (elastómero) y de vías (pavimento asfáltico envejecido).

\begin{tabular}{|c|c|c|c|}
\hline Tratamientos & Flujo in/100 & $\begin{array}{c}\text { \% de vacíos en agregados } \\
\text { minerales }\end{array}$ & $\begin{array}{c}\% \text { de vacíos en rellenos } \\
\text { de asfalto }\end{array}$ \\
\hline Factor G & * & $* *$ & $* *$ \\
\hline G1 granulometría fina & $14,83(a b)$ & $15,12 \%(b)$ & $71,80 \%(a)$ \\
\hline G2 granulometría media & $14,42(a)$ & $15,40 \%(b)$ & $70,36 \%(a b)$ \\
\hline G3 granulometría gruesa & $15,17(b)$ & $15,82 \%(a)$ & $68,47 \%(b)$ \\
\hline Factor $\mathrm{E}$ & $*$ & NS & NS \\
\hline E1 4\% elastómero & $14,44(a)$ & $15,57 \%$ & $69,66 \%$ \\
\hline E2 8\% elastómero & $14,56(a b)$ & $15,39 \%$ & $70,17 \%$ \\
\hline E3 12\% elastómero & $14,89(a b)$ & $15,33 \%$ & $70,96 \%$ \\
\hline E4 16\% elastómero & $15,33(b)$ & $15,49 \%$ & $70,14 \%$ \\
\hline Interacción & NS & NS & NS \\
\hline G1E1 & 14,67 & $15,12 \%$ & $72,57 \%$ \\
\hline G1E2 & 14,33 & $15,03 \%$ & $71,67 \%$ \\
\hline G1E3 & 15,00 & $15,22 \%$ & $70,59 \%$ \\
\hline G1E4 & 15,33 & $15,10 \%$ & $72,67 \%$ \\
\hline G2E1 & 14,00 & $15,67 \%$ & $68,13 \%$ \\
\hline G2E2 & 14,00 & $15,36 \%$ & $71,27 \%$ \\
\hline G2E3 & 14,67 & $15,15 \%$ & $72,47 \%$ \\
\hline G2E4 & 15,00 & $15,43 \%$ & $69,55 \%$ \\
\hline G3E1 & 14,67 & $15,92 \%$ & $68,29 \%$ \\
\hline G3E2 & 15,33 & $15,78 \%$ & $67,57 \%$ \\
\hline G3E3 & 15,00 & $15,63 \%$ & $69,83 \%$ \\
\hline G3E4 & 15,67 & $15,94 \%$ & $68,18 \%$ \\
\hline Factorial versus testigo & ** & NS & NS \\
\hline Testigo absoluto & $13,00 \mathrm{a}$ & $14,96 \%$ & $71,92 \%$ \\
\hline Granulometría & $14,81 \mathrm{~b}$ & $15,45 \%$ & $70,21 \%$ \\
\hline Elastómero & $14,81 \mathrm{~b}$ & $15,59 \%$ & $70,23 \%$ \\
\hline Coeficiente de variación & 4,42 & 2,57 & 3,04 \\
\hline $\mathbf{P}$ & 0,0001 & 0,041 & 0,038 \\
\hline
\end{tabular}

$\left.{ }^{*}\right)$ Diferencia significativa entre los tratamientos.

$\left.{ }^{(* *}\right)$ Diferencia altamente significativa entre los tratamientos. Letras en común no son estadísticamente diferentes.

Fuente: Elaboración propia.

dos rangos de similitud estadística, donde destacó el factor $\mathrm{G} 3$ con $15,82 \%$ de vacíos en agregados minerales, mientras que $\mathrm{G} 1$ y $\mathrm{G} 2$ tuvieron $15,40 \%$ y $15,12 \%$, respectivamente, y se obtuvieron valores bajos en "\% de vacíos en agregados minerales". Las exigencias del Ministerio de Transporte y Obras Públicas del Ecuador (2002) no establecen valores máximos a considerar, por ende, es errado afirmar que cuanto mayor sean los vacíos en agregados minerales (VAM) más espacio habrá disponible para las películas de asfalto. De tal forma, según Carrasco (2004):

Existen valores mínimos para VMA [o VAM] los cuales están recomendados y especificados como función del tamaño del agregado. Estos valores se basan en el hecho de que cuanta más gruesa sea la película de asfalto que cubre las partículas de agregado, más durable será la mezcla (pp. 62-63).

6. Vacíos en rellenos de asfalto. En la Tabla 9 se observan los valores promedios de la variable "\% de vacíos en rellenos de asfalto"; el análisis de varianza no determinó diferencias estadísticas para el factor $E$, interacción GxE y comparación factorial versus testigo; sin embargo, se evidenciaron diferencias estadísticas para el factor $\mathrm{G}$. En la prueba de significancia realizada al factor $G$ se establecieron dos rangos de similitud estadística, en el cual destacó $\mathrm{G} 3$ con $68,47 \%$, seguido de $\mathrm{G} 2$ con $70,36 \%$ y, por último, G1 con $71,80 \%$. De esta manera, los resultados coinciden con las exigencias del diseño de Marshall, el cual establece un valor mínimo de $65 \%$ y el máximo de $75 \%$ (Ministerio de Transporte y Obras Públicas del Ecuador, 2002). 


\section{CONCLUSIONES}

1. La adición de $4 \%, 8 \%$ y $12 \%$ de elastómero en el diseño de mezclas asfálticas con pavimento asfáltico envejecido derivó en un buen proceder, lo que sirve para ser utilizado a nivel de campo en mezclas asfálticas modificadas.

2. Las granulometrías media y fina, en el diseño de mezclas asfálticas con pavimento asfáltico envejecido, contribuyeron a obtener una buena conducta con la reutilización del elastómero y del pavimento asfáltico, por lo tanto, puede ser utilizado a nivel de campo en mezclas asfálticas modificadas.

\section{REFERENCIAS BIBLIOGRÁFICAS}

[1] Amorim, E. y Lima, L. (2018). Uso de residuos de caucho en pavimentos de asfalto: una revisión de literatura. Revista científica multidisciplinaria base de conocimiento, 3(2), 39-47.

[2] Bertalot, E. (2017). Una mirada al reciclaje de neumáticos fuera de uso. Revista SLTCaucho, (19), 18-19.

[3] Bureau of International Recycling (BIR) (2016). Neumáticos. Recuperado de http://www.bir. org/industry-es-es/tyres-es-es/

[4] Castro, V. (2015). Estudio de factibilidad de creación de una empresa recicladora de neumáticos desechados, para la producción de caucho modificador de asfalto, en la provincia de Guayas (Tesis de maestría). Universidad de Guayaquil, Ecuador.

[5] Centro de Estudios y Experimentación de Obras Públicas (CEDEX) (2011). Reciclado de pavimentos asfálticos. Madrid, España: Centro de Estudios y Experimentación de Obras Públicas.

[6] Figueroa, A., Fonseca, E., Amaya, C. y Prieto, M. (2008). Contrastación entre el asfalto modificado con poliestireno y llanta triturada empleando dos métodos de mezclado. Revista Épsilon, (10), 67-79.

[7] Garnica, P., Delgado, H., Gómez, J., Alonso, S. y Alarcón, H. (2004). Aspectos del diseño volumétrico de mezclas asfálticas. Sanfandila, México: Secretaría de Comunicaciones y Transportes / Instituto Mexicano del Transporte.

[8] Grytsenko, A., Pozdniakova, Y. y Vnukova, N. (2015). Estimation of possibility of tyre utilization products usage as alternative fuel. Recuperado de https://dspace.khadi.kharkov.ua/dspace/ bitstream/123456789/1082/1/\%D0\%90\%D0\% A2_36_07.pdf

[9] Lacamara, M. (2014). Brasil estrena el asfalto ecológico. Twenergy. Recuperado de https:// twenergy.com/a/brasil-estrena-el-asfaltoecológico

[10] Lecitra, M. (2010). Reducir, reutilizar y reciclar: el problema de los residuos sólidos urbanos. Recuperado de https://geic.files.wordpress. com/2010/10/reducir-reutilizar-y-reciclar.pdf

[11] Martín, A. (2015). Aplicación del caucho reciclado como solución constructiva ecológica. Recuperado de https://riunet.upv.es/bitstream/ handle/10251/55735/MART\%C3\%8DN\%20 -\%20Aplicaci\%C3\%B3n\%20del\%20caucho\%20 reciclado $\% 20$ como $\% 20$ soluci\%C3\%B3n $\% 20$ constructiva $\% 20$ e col \% C $3 \%$ B 3 gica . pdf?sequence $=1$

[12] Ministerio de Transporte y Obras Públicas (MTOP) (2002). Especificaciones generales para la construcción de caminos y puentes. Quito, Ecuador: Ministerio de Obras Públicas / República del Ecuador.

[13] Paiva, G y Ramos, G. (2014). Reciclado de pavimentos asfálticos y su reutilización para el diseño de mezcla de asfalto en caliente (Tesis de licenciatura). Universidad Señor de Sipán, Perú.

[14] Peláez, G., Velásquez, S. y Giraldo, D. (2017). Aplicaciones de caucho reciclado: una revisión de la literatura. Revista Ciencia e Ingeniería Neogranadina, 27(2), 27-50.

[15] Sánchez, R. (2012). Segunda vida de los neumáticos usados. Revista Química Viva, 11(1), 25-40.

[16] Carrasco, D. (2004). Estudio comparativo entre mezclas asfálticas en caliente y mezclas asfálticas con emulsiones tibias. (Tesis de licenciatura). Universidad de Piura, Perú. 


\section{Design of asphalt mixtures integrating solid waste from the automotive industry (elastomer) and roadways (aged asphalt pavement) in Manabí, Ecuador}

Gabriel Antonio Navarrete Schettini ${ }^{1}$

Recelved: 11/05/2018 AcCepted: 05/12/2018

\begin{abstract}
The purpose of this study is to design asphalt mixtures by integrating solid waste from used tires (elastomer) and aged asphalt pavement in Bolivar Canton, Manabi Province, Ecuador. The factors studied were 4\%, $8 \%$, $12 \%$ and $16 \%$ elastomer, and fine, average and coarse grading, using $52 \%$ aged asphalt pavement. A completely randomized two-way factorial arrangement, $\mathrm{GxE}+1$, with thirteen treatments and three replications was used where $G$ corresponds to grading of aggregates used and $E$ to elastomer. On the basis of the positive outcome of the design of asphalt mixtures with aged asphalt pavement, considering the addition of $4 \%, 8 \%$ and $12 \%$ of elastomer in the average and fine grading curves, it was concluded that the design can be used in light and medium traffic roads, such as those in Bolivar Canton, Ecuador.
\end{abstract}

Keywords: Elastomer; aged asphalt pavement; industrial waste.

\section{INTRODUCTION}

There are an estimated 800 million commercial vehicles in use worldwide, and almost 70 million are added to that number every year (Bureau of International Recycling, 2016). Moreover, the global consumption of elastomer in 2014 was estimated at 28.9 million tons, increasing by $0.7 \%$ in 2015 according to Pélaez, Velásquez and Giraldo (2017). From that global quantity, in "Ecuador cada año se desechan alrededor de 2.4 millones de neumáticos" [Ecuador, an estimated 2.4 million tires are discarded every year] (Castro, 2015, p. 2), and being a product designed to offer the greatest possible resistance to wear during use, it is estimated that their natural decomposition will occur over a period longer than 600 years. The composition of tires includes dangerous components such as lead, chromium, cadmium and other heavy metals that represent a threat to both health and environment when improperly disposed of (Bertalot, 2017). The recycling of asphalt pavement is carried out on deteriorated materials that have largely lost their initial properties. The recovery of milled materials through recycling, later enriched by adding stone materials, is technically feasible (Centro de Estudios y Experimentación de Obras Públicas, 2011).

What is more, Sánchez (2012) states that "los materiales que se obtienen tras el tratamiento de los residuos de neumáticos, una vez separados los restos aprovechables en la industria, pueden [tener varios usos]' [the materials obtained through processing of scrap tires, once the industrially usable materials have been separated, can [have several uses]] (p. 29). Scholars such as Sánchez (2012), Martín (2015) and Grytsenko, Pozdniakova and Vnukova (2015) agree that one of the uses of rubber is to serve as a component of the asphalt layers that are used in road construction, thus reducing the extraction of aggregates from quarries. Furthermore, the material obtained confers special characteristics to asphalt layers, it can be used as rubber in the wearing course in order for it to have a longer average lifespan, more elasticity, fewer deformations, more crack resistance and more skid resistance. In addition to the above, it also has the char-

1 Civil engineer from the Universidad Central del Ecuador and Master of Education and Social Development from the Universidad Tecnológica Equinoccial. Currently, Professor at the Escuela Superior Politécnica Agropecuaria de Manabí Manuel Félix López (ESPAM MFL). Manabí, Ecuador.

E-mail: gnavarrete@espam.edu.ec 
acteristic of being permeable concrete, which provides the asphalt mixture features to prevent water accumulation, increasing as a result its adherence, preventing standing water formation and conferring good visual conditions and low noise level. It provides therefore both environmental and economic benefits, as the roads become more resistant, durable and less fragile as a result of the incorporation of rubber. It even helps prevent roads from breaking and has a 10-year lifespan. (Amorim \& Lima, 2018).

At the same time, Lacamara (2014) provides relevant data which demonstrates that "se pueden emplear entre 1000 y 7000 neumáticos por kilómetro de carretera de dos carriles. Cifras tan elevadas colocan el pavimento asfáltico como una de las grandes soluciones para emplear los neumáticos fuera de uso" [between 1000 and 7000 tires can be used per kilometer of two-lane road. Such high numbers make asphalt pavement one of the best options to use scrap tires] (para. 13). Thus, pavement recycling is a more competitive and sustainable pavement rehabilitation alternative, because it helps to minimize the usage of non-renewable resources, natural aggregates and asphalt, while it also prevents the creation of waste and filling of landfills (Paiva \& Ramos, 2014). Likewise, it also includes a series of construction techniques for the comprehensive use of aged road surface and pavement materials. For these reasons, it is of vital importance to develop a sustainable opportunity for people whose

[...] basan sus ingresos en la recuperación informal de lo que otros desechan, están expuestos a peligros [sanitarios] [...]. Dentro de este sector informal, es importante el número de niños y niñas que encuentran en el trabajo de recuperación de residuos la única forma de sobrevivir ante una sociedad muchas veces indiferente [income is based on the informal recovery of other people's waste, and who are exposed to [health] hazards (...). Within this informal sector, the number of children who find in waste collection the only way to survive in an often indifferent society is significant] (Lecitra, 2010, p. 4).

Thus, it is proposed to design asphalt mixtures by integrating solid waste obtained from scrap tires (elastomer) and aged asphalt pavement in Bolívar Canton, Manabí Province, Ecuador.

\section{METHODOLOGY}

1. Experimental design. A completely randomized two-way factorial arrangement, GxE+1, with thirteen treatments and three replications was used, where $G$ corresponds to grading of aggregates used and $E$ to elastomer. InfoStad software was used to determine the coefficient of variation (CV\%) and Tukey test at $5 \%$ error probability was used for variables where statistical differences were found.

2. Dosing of aggregates. The dosing and tolerance of materials were determined according to the values shown in Table 1, which are recommended by the general specifications for road and bridge construction (Ministerio de Transportes y Obras Públicas del Ecuador, 2002).

3. Grading of materials. An abrasion test was conducted on $1 / 2$ " material using gradation $B$, which

Table 1. General specifications for road and bridge construction

\begin{tabular}{|c|c|c|c|c|}
\hline \multirow[t]{2}{*}{ Sieve } & \multicolumn{4}{|c|}{ Percent by weight passing the square mesh sieves } \\
\hline & No. $3 / 4$ & No. $1 / 2$ & No. $3 / 8$ & No. 4 \\
\hline No. $1 / 2(25.4 \mathrm{~mm})$ & 100 & & & \\
\hline No. 3/4 $(19.0 \mathrm{~mm})$ & $90-100$ & 100 & & \\
\hline No. $1 / 2(12.7 \mathrm{~mm})$ & & $90-100$ & 100 & \\
\hline No. $3 / 8(9.50 \mathrm{~mm})$ & $56-80$ & & $90-100$ & 100 \\
\hline No. $4(4.75 \mathrm{~mm})$ & $35-65$ & $44-74$ & $55-85$ & $80-100$ \\
\hline No. $8(2.36 \mathrm{~mm})$ & $23-49$ & $28-58$ & $32-67$ & $65-100$ \\
\hline No. $16(1.18 \mathrm{~mm})$ & & & & $40-80$ \\
\hline No. $30(0.60 \mathrm{~mm})$ & & & & $25-65$ \\
\hline No. $50(0.30 \mathrm{~mm})$ & $5-19$ & $5-21$ & $7-23$ & $7-40$ \\
\hline No. $100(0.15 \mathrm{~mm})$ & & & & $3-20$ \\
\hline No. $200(0.075 \mathrm{~mm})$ & $2-8$ & $2-10$ & $2-10$ & $2-10$ \\
\hline
\end{tabular}

Source: Ministry of Transport and Public Works of Ecuador (2002). 
consists on obtaining material extracted from the grading, using $2500 \mathrm{~g} \pm 2$ passing $19 \mathrm{~mm}$ or No. 3/4 sieve, retained on $12.5 \mathrm{~mm}$ or No. $1 / 2$ sieve, and $2500 \mathrm{~g} \pm 2$ passing $12.5 \mathrm{~mm}$ or No. $1 / 2$ sieve, retained on $9.5 \mathrm{~mm}$ or No. 3/8 sieve. The sample was placed in the Los Angeles Abrasion machine and rotated for 500 revolutions. After the revolutions, the material was discharged from the machine, sieved through $1.70 \mathrm{~mm}$ or No. 12 sieve, washed and dried at $110{ }^{\circ} \mathrm{C}$. After drying the material, it was weighed to calculate the degradation coefficient.

The unit weight or density test consisted on having completely dried materials $(1 / 2,3 / 8$, broken coal and sand), and metal molds with known weights and volumes to perform the test, which consists of constantly adding the material into the recipient until it is full, levelling and then weighing it. This procedure was repeated three times in order to determine loose density. In contrast, to determine compacted density, the container was filled with three equal layers of material and each layer was rodded 25 times. Once testing was completed for 1/2" (aggregate 1), 3/8" (aggregate 2), broken coal (aggregate 3 ) and sand (aggregate 4) materials, data entry and results calculation were performed, as shown in Table 2.

\section{Determination of the optimum percentage} of asphalt. The optimum percentage of asphalt is obtained by using the voids percentage, which obtained a result of $6.2 \%$, as observed in Table 3 . Additionally, other results were obtained from the curves, such as unit weights (density), voids, stability, voids in mineral aggregate (VMA), flow and voids filled with asphalt (VFA).

5. Integration of aged asphalt pavement and elastomer. The molding and testing of the test briquettes was performed in a similar manner, with the addition of crumb rubber, material that passes No. 10 sieve and is retained by No. 40 sieve, and aged asphalt pavement. Stone aggregates were mixed individually without the addition of solid waste, maintaining maximum and minimum temperatures of 160 and $80{ }^{\circ} \mathrm{C}$. According to Figueroa, Fonseca, Amaya, and Prieto (2008), this temperature produces a better blending process, achieving a better homogenization of modified asphalt binder. Table 4 shows the average values of the Marshall Method requirements, where $52 \%$ of aged asphalt

Table 2. Aggregates mixture

\begin{tabular}{|c|c|c|c|c|c|c|c|}
\hline \multicolumn{8}{|c|}{ Combined grading for the mixture } \\
\hline \multirow{2}{*}{ Type of material } & \multirow{2}{*}{$\begin{array}{l}\text { Percentage for } \\
\text { mixture }\end{array}$} & \multicolumn{6}{|c|}{ Percentage passing sieve } \\
\hline & & No. $3 / 4$ & No. $1 / 2$ & No. 4 & No. 8 & No. 50 & No. 200 \\
\hline Aggregate No. 1 & $12 \%$ & 12.0 & 6.2 & 0.3 & 0.3 & 0.2 & 0.2 \\
\hline Aggregate No. 2 & $28 \%$ & 28.0 & 28.0 & 4.9 & 1.1 & 0.9 & 0.7 \\
\hline Aggregate No. 3 & $45 \%$ & 45.0 & 45.0 & 42.5 & 27.5 & 12.4 & 5.7 \\
\hline Aggregate No. 4 & $15 \%$ & 15.0 & 15.0 & 14.9 & 14.5 & 4.4 & 0.2 \\
\hline \multicolumn{2}{|c|}{ Obtained grading } & 100.0 & 94.2 & 62.5 & 43.3 & 18.0 & 6.8 \\
\hline \multicolumn{2}{|c|}{ Specification } & 100 & $90-100$ & $44-77$ & $28-58$ & $5-21$ & $2-10$ \\
\hline \multicolumn{2}{|c|}{ Expected grading } & 100.0 & 95.0 & 60.5 & 43.0 & 13.0 & 6.0 \\
\hline
\end{tabular}

Source: Prepared by the author.

Table 3. Marshall Method to obtain the optimum percentage of asphalt.

\begin{tabular}{|l|l|l|l|}
\hline Properties & $\%$ asphalt-design & Obtained value & Requirements \\
\hline Stability & 6.20 & 2546 & $>1800 \mathrm{lbs}$. \\
\hline Flow & 6.20 & 12.0 & $8-16$ in/100 \\
\hline Unit weight & 6.20 & 2281 & $<2000$ \\
\hline Voids in mixture & 6.20 & 4.0 & $3.0-5.0 \%$ \\
\hline Voids in mineral aggregates & 6.20 & 15.20 & $>13 \%$ \\
\hline Voids filled with asphalt & 6.20 & 71 & $65-75 \%$ \\
\hline
\end{tabular}

Source: Prepared by the author. 
pavement was used and it was determined that the asphalt pavement did not meet the requirements suggested by the Ministry of Transport and Public Works of Ecuador (2002) and Garnica, Delgado, Gómez, Alonso and Alarcón (2004), for which reason stone aggregates were added.

Tables 5, 6 and 7 show grading with $52 \%$ of aged asphalt pavement enriched with stone aggregates.
Once the optimum percentage of asphalt in the mixture was determined to be $6.2 \%$, different percentages of crumb rubber, from 4 to $16 \%$, were added via dry mixing.

6. Variables to be evaluated in asphalt mixtures. The Marshall Method was used to evaluate asphalt mixtures. Bulk density, stability, flow, voids in mixture, voids in mineral aggregates and voids filled

Table 4. Marshall Method of aged asphalt pavement.

\begin{tabular}{|c|c|c|c|}
\hline Properties & \% asphalt-design & Obtained value & Requirements \\
\hline Stability & 5.70 & 3381 & $>1800 \mathrm{lbs}$. \\
\hline Flow & 5.70 & 15.5 & $8-16 \mathrm{in} / 100$ \\
\hline Unit weight & 5.70 & 2174 & $>2000$ \\
\hline Voids in mixture & 5.70 & 6.37 & $3.0-5.0 \%$ \\
\hline Voids in aggregates & 5.70 & 19.62 & $>13 \%$ \\
\hline Voids filled with asphalts & 5.70 & 67.37 & $65-75 \%$ \\
\hline
\end{tabular}

Source: Prepared by the author.

Table 5. Middle limit gradation of aged asphalt pavement enriched with stone aggregates

\begin{tabular}{|c|c|c|c|c|c|c|c|}
\hline \multicolumn{8}{|c|}{ Combined gradation for the mixture } \\
\hline \multirow{2}{*}{ Type of material } & \multirow{2}{*}{$\begin{array}{l}\text { Percentage for } \\
\text { mixture }\end{array}$} & \multicolumn{6}{|c|}{ Percentage passing sieve } \\
\hline & & $N .^{\circ} 3 / 4$ & $N .{ }^{\circ} 1 / 2$ & N. ${ }^{\circ} 4$ & N. ${ }^{\circ} 8$ & N. ${ }^{\circ} 50$ & N. ${ }^{\circ} 200$ \\
\hline Aggregate No. 1 & $10 \%$ & 10.0 & 4.8 & 0.2 & 0.2 & 0.1 & 0.1 \\
\hline Aggregate No. 2 & $16 \%$ & 16.0 & 16.0 & 2.3 & 0.7 & 0.5 & 0.4 \\
\hline Aggregate No. 3 & $16 \%$ & 16.0 & 16.0 & 15.3 & 10.2 & 4.8 & 2.0 \\
\hline Aggregate No. 4 & $6 \%$ & 6.0 & 6.0 & 5.9 & 5.8 & 1.7 & 0.1 \\
\hline Recycled & $52 \%$ & 52.0 & 50.4 & 37.7 & 25.8 & 10.5 & 2.0 \\
\hline \multicolumn{2}{|c|}{ Obtained grading } & 100.0 & 93.2 & 61.4 & 42.7 & 17.6 & 4.6 \\
\hline \multicolumn{2}{|c|}{ Specification } & 100 & $90-100$ & 44-77 & $28-58$ & $5-21$ & $2-10$ \\
\hline \multicolumn{2}{|c|}{ Expected grading } & 100.0 & 95.0 & 60.5 & 43.0 & 13.0 & 6.0 \\
\hline
\end{tabular}

Source: Prepared by the author.

Table 6. Fine gradation of aged asphalt pavement enriched with stone aggregates

\begin{tabular}{|c|c|c|c|c|c|c|c|}
\hline \multicolumn{8}{|c|}{ Combined gradation for the mixture } \\
\hline \multirow{2}{*}{ Type of material } & \multirow{2}{*}{$\begin{array}{l}\text { Percentage for } \\
\text { mixture }\end{array}$} & \multicolumn{6}{|c|}{ Percentage passing sieve } \\
\hline & & No. $3 / 4$ & No. $1 / 2$ & No. 4 & No. 8 & No. 50 & No. 200 \\
\hline Aggregate No. 1 & $8 \%$ & 8.0 & 3.8 & 0.1 & 0.1 & 0.1 & 0.1 \\
\hline Aggregate No. 2 & $8 \%$ & 8.0 & 8.0 & 1.2 & 0.3 & 0.3 & 0.2 \\
\hline Aggregate No. 3 & $26 \%$ & 26.0 & 26.0 & 24.9 & 16.6 & 7.8 & 3.3 \\
\hline Aggregate No. 4 & $6 \%$ & 6.0 & 6.0 & 5.9 & 5.8 & 1.7 & 0.1 \\
\hline Recycled & $52 \%$ & 5.0 & 50.4 & 37.7 & 25.8 & 10.5 & 2.0 \\
\hline \multicolumn{2}{|c|}{ Obtained grading } & 100.0 & 94.2 & 69.8 & 48.8 & 20.3 & 5.6 \\
\hline \multicolumn{2}{|c|}{ Specification } & 100 & $90-100$ & $44-77$ & $28-58$ & $5-21$ & $2-10$ \\
\hline \multicolumn{2}{|c|}{ Expected grading } & 100.0 & 95.0 & 60.5 & 43.0 & 13.0 & 6.0 \\
\hline
\end{tabular}

Source: Prepared by the author. 
Table 7. Coarse gradation of aged asphalt pavement enriched with stone aggregates

\begin{tabular}{|c|c|c|c|c|c|c|c|}
\hline \multicolumn{8}{|c|}{ Combined gradation for the mixture } \\
\hline \multirow{2}{*}{ Type of material } & \multirow{2}{*}{$\begin{array}{l}\text { Percentage for } \\
\text { mixture }\end{array}$} & \multicolumn{6}{|c|}{ Percentage passing sieve } \\
\hline & & No. $3 / 4$ & No. $1 / 2$ & No. 4 & No. 8 & No. 50 & No. 200 \\
\hline Aggregate No. 1 & $12 \%$ & 12.0 & 5.7 & 0.2 & 0.2 & 0.2 & 0.1 \\
\hline Aggregate No. 2 & $22 \%$ & 20.0 & 22.0 & 3.2 & 0.9 & 0.7 & 0.5 \\
\hline Aggregate No. 3 & $14 \%$ & 14.0 & 14.0 & 13.4 & 9.0 & 4.2 & 1.8 \\
\hline Aggregate No. 4 & $2 \%$ & 2.0 & 2.0 & 2.0 & 1.9 & 0.6 & 0.0 \\
\hline Recycled & $52 \%$ & 52.0 & 50.4 & 37.7 & 25.8 & 10.5 & 2.0 \\
\hline \multicolumn{2}{|c|}{ Obtained grading } & 100.0 & 94.1 & 56.5 & 37.8 & 16.1 & 4.5 \\
\hline \multicolumn{2}{|c|}{ Specification } & 100 & $90-100$ & 44-77 & $28-58$ & $5-21$ & $2-10$ \\
\hline \multicolumn{2}{|c|}{ Expected grading } & 100.0 & 95.0 & 60.5 & 43.0 & 13.0 & 6.0 \\
\hline
\end{tabular}

Source: Prepared by the author.

with asphalt were determined in accordance with the Manual de Especificaciones [Specifications Manual] (Ministerio de Transporte y Obras Públicas del Ecuador, 2002 and Garnica et al., 2004).

\section{RESULTS AND DISCUSSION}

1. Bulk density. The average values of variable "Bulk density" are shown in Table 8. The analysis of variance (ANOVA) did not determine statistical differences for factor $G$, factor $E$, interaction $G x E$, nor for the contrast all treatments vs. control.

2. Percentage of voids in mixture. The average values of variable "\% of voids in mixture" are shown in Table 8. No statistical differences were determined for factor $E$, interaction GxE, nor for the contrast all treatments vs. control using the analysis of variance (ANOVA); however, it did determine statistics for factor $\mathrm{G}$. The significance test determined two statistical similarities in factor $\mathrm{G}$, where the best "\% of voids in mixture" corresponded to $\mathrm{G} 1$ with $4.26 \%$, followed by G2. In this regard, the Ministry of Transport and Public Works of Ecuador (2002) states that the maximum voids percentage value required is $5 \%$; therefore, the obtained coarse grading equal to $5 \%$ does not comply with the requirements. It should be noted that the permissible voids percentage in laboratory samples for base and surface layers are between $3 \%$ and $5 \%$.

3. Stability. The average values of variable "Stability" are shown in Table 8. The analysis of variance (ANOVA) determined statistical differences for factor $\mathrm{G}$, factor $\mathrm{E}$, interaction $\mathrm{GxE}$, and for contrast all treatments vs. control. Significance test performed on factor $\mathrm{G}$ determined two statistical similarity features, where $\mathrm{G} 2$ obtained the highest stability with 2880.86 lbs., whereas G3 obtained the lowest with 2640.34 lbs. Likewise, significance test performed on factor $\mathrm{E}$ determined three statistical similarities, where E3 obtained the highest stability with 3018.59 lbs., whereas E4 obtained the lowest with 2472.87 lbs. Significance test performed on interaction GxE determined four statistical similarities, where G2G3 (average G-12\% elastomer) and G1G3 (fine-12\% elastomer) obtained $3119.73 \mathrm{lbs}$. and $3042.36 \mathrm{lbs}$., respectively. Furthermore, G2G3 and G1G3 obtained the highest stability, whereas G3G4 (coarse G-16\% elastomer) obtained the lowest stability with $2310.61 \mathrm{lbs}$. In the contrast all treatments vs. control, three statistical similarities were established, where factor $\mathrm{G}$ stood out with $2790.10 \mathrm{lbs}$., followed by factor $E$ with $2640.10 \mathrm{lbs}$., and, lastly, control with 2605.67 lbs. of stability.

Un pavimento estable es capaz de mantener su forma y lisura bajo cargas repetitivas [...] Las especificaciones de estabilidad deben ser lo suficiente altas para acomodar adecuadamente el tránsito esperado, pero no más altas de lo que exijan las condiciones de transito [sic] [A stable pavement is able to maintain its shape and smoothness under repeated traffic loads (...) Stability specifications must be high enough to properly accommodate expected traffic, but not higher than required by traffic conditions [sic]](Carrasco, 2004, p. 65).

In addition, the Ministry of Transport and Public Works of Ecuador (2002) requires stability ratings above $1800 \mathrm{lbs}$., to ensure that all treatments comply with the requirements of the Marshall Method.

4. Flow. The average values of variable "Flow" are shown in Table 9. No statistical differences were determined for interaction GxE using the analysis of variance (ANOVA), instead, statistical differences for factor $G$, factor $E$, and contrast all treatments vs. control were observed. Significance test conducted 
Table 8. Bulk density, percentage of voids in mixtures and stability. Design of asphalt mixtures integrating solid waste from the automotive industry (elastomer) and roads (aged asphalt pavement).

\begin{tabular}{|c|c|c|c|}
\hline Treatments & Bulk density & $\%$ voids in mixture & Stability \\
\hline Factor $\mathbf{G}$ & NS & ** & $* *$ \\
\hline G1 fine grading & 2282.17 & $4.26 \%(a)$ & 2849.09 lbs. (a) \\
\hline G2 average grading & 2274.67 & $4.58 \%(a b)$ & 2880.86 lbs. (a) \\
\hline G3 coarse grading & 2272.50 & $5.00 \%(b)$ & 2640.34 lbs. (b) \\
\hline Factor $\mathrm{E}$ & NS & NS & $* *$ \\
\hline E1 $4 \%$ of elastomer & 2270.11 & $4.73 \%$ & 2785.78 lbs. (b) \\
\hline E2 $8 \%$ of elastomer & 2274.89 & $4.60 \%$ & 2883.15 lbs. (b) \\
\hline E3 $12 \%$ of elastomer & 2276.56 & $4.46 \%$ & 3018.59 lbs. (a) \\
\hline E4 $16 \%$ of elastomer & 2284.22 & $4.64 \%$ & 2472.87 lbs. (c) \\
\hline Interaction & NS & NS & ** \\
\hline G1E1 & 2282 & $4.15 \%$ & 2890.00 lbs. (ab) \\
\hline G1E2 & 2284.67 & $4.27 \%$ & 2953.57 lbs. (ab) \\
\hline G1E3 & 2279.33 & $4.49 \%$ & 2042.36 lbs. (a) \\
\hline G1E4 & 2282.67 & $4.13 \%$ & 2510.42 lbs. (de) \\
\hline G2E1 & 2267.33 & $5.00 \%$ & 2870.94 lbs. (abc) \\
\hline G2E2 & 2275.67 & $4.42 \%$ & 2935.18 lbs. (ab) \\
\hline G2E3 & 2281.67 & $4.18 \%$ & 3119.73 lbs. (a) \\
\hline G2E4 & 2274 & $4.72 \%$ & 2597.57 lbs. (cd) \\
\hline G3E1 & 2261 & $5.05 \%$ & 2596.39 lbs. (cd) \\
\hline G3E2 & 2264.33 & $5.12 \%$ & 2760.70 lbs. (bcd) \\
\hline G3E3 & 2268.67 & $4.72 \%$ & 2893.68 lbs. (ab) \\
\hline G3E4 & 2296 & $5.07 \%$ & 2310.61 lbs. (e) \\
\hline All treatments vs control & NS & NS & $* *$ \\
\hline Absolute control sample & 2286.67 & $4.21 \%$ & 2605.67 lbs. (c) \\
\hline Grading & 2278.11 & $4.61 \%$ & 2790.10 lbs. (a) \\
\hline Elastomer & 2290.30 & $4.60 \%$ & 2640.10 lbs. (b) \\
\hline Coefficient of variation & 0.87 & 9.75 & 3.38 \\
\hline $\mathbf{P}$ & 0.6711 & 0.044 & $<0.0001$ \\
\hline
\end{tabular}

$\left({ }^{*}\right)$ Significant difference between treatments.

$\left.{ }^{* *}\right)$ Highly significant difference between treatments. Common letters are not statistically different.

Source: Prepared by the author.

on factor $G$ determined two statistical similarities, where G2 and G1 obtained 14.42 in/100 and 14.83 in/100, respectively and stood out in the coarse grading with $15.17 \mathrm{in} / 100$. Statistical test conducted on factor $E$ determined two statistical similarity features, where E1, E2 and E3 obtained 14.44 in/100, $14.56 \mathrm{in} / 100$ and $14.89 \mathrm{in} / 100$ respectively and stood out with good flow values compared to the $15.33 \mathrm{in} / 100$ obtained by E4. Significance test conducted on contrast all treatments vs. control determined two statistical similarity features, where the control obtained the best flow results with $13 \mathrm{in} / 100$, followed by factors $G$ and $E$ both with $14.81 \mathrm{in} / 100$. Thus, asphalt mixtures with reusable material only comply with the requirements established for light and medium traffic. This is consistent with the requirements set out by the Ministry of Transport and
Public Works of Ecuador (2002) that established a minimum of $8 \mathrm{in} / 100$ for all kinds of traffic, such as light, medium, heavy and very heavy traffic. The maximum flow value established for heavy and very heavy traffic is $14 \mathrm{in} / 100$, whereas the maximum for medium and light traffic is $16 \mathrm{in} / 100$.

5. Voids in mineral aggregates. The values of variable "\% of voids in mineral aggregates" are shown in Table 9. No significant statistical differences were determined for factor $\mathrm{E}$, interaction $\mathrm{GxE}$ nor for contrast all treatments vs. control using the analysis of variance (ANOVA); however, statistical differences were determined for factor $\mathrm{G}$. Significance test conducted on factor $\mathrm{G}$ determined two statistical similarities, where factor G3 stood out with $15.82 \%$ of voids in mineral aggregates, whereas G1 and G2 
Table 9. Flow, voids in mineral aggregates and voids filled with asphalt. Design of asphalt mixtures integrating solid waste from the automotive (elastomer) and roads (aged asphalt pavement) industry.

\begin{tabular}{|c|c|c|c|}
\hline Treatments & Flow in/100 & $\%$ voids in mineral aggregate & $\%$ voids filled with asphalt \\
\hline Factor $\mathbf{G}$ & * & ** & ** \\
\hline G1 fine grading & $14.83(a b)$ & $15.12 \%(b)$ & $71.80 \%(a)$ \\
\hline G2 average grading & $14.42(\mathrm{a})$ & $15.40 \%(b)$ & $70.36 \%(a b)$ \\
\hline G3 coarse grading & $15.17(b)$ & $15.82 \%(a)$ & $68.47 \%(b)$ \\
\hline Factor E & * & NS & NS \\
\hline E1 $4 \%$ of elastomer & $14.44(\mathrm{a})$ & $15.57 \%$ & $69.66 \%$ \\
\hline E2 $8 \%$ of elastomer & $14.56(a b)$ & $15.39 \%$ & $70.17 \%$ \\
\hline E3 $12 \%$ of elastomer & $14.89(\mathrm{ab})$ & $15.33 \%$ & $70.96 \%$ \\
\hline E4 $16 \%$ of elastomer & $15.33(b)$ & $15.49 \%$ & $70.14 \%$ \\
\hline Interaction & NS & NS & NS \\
\hline G1E1 & 14.67 & $15.12 \%$ & $72.57 \%$ \\
\hline G1E2 & 14.33 & $15.03 \%$ & $71.67 \%$ \\
\hline G1E3 & 15.00 & $15.22 \%$ & $70.59 \%$ \\
\hline G1E4 & 15.33 & $15.10 \%$ & $72.67 \%$ \\
\hline G2E1 & 14.00 & $15.67 \%$ & $68.13 \%$ \\
\hline G2E2 & 14.00 & $15.36 \%$ & $71.27 \%$ \\
\hline G2E3 & 14.67 & $15.15 \%$ & $72.47 \%$ \\
\hline G2E4 & 15.00 & $15.43 \%$ & $69.55 \%$ \\
\hline G3E1 & 14.67 & $15.92 \%$ & $68.29 \%$ \\
\hline G3E2 & 15.33 & $15.78 \%$ & $67.57 \%$ \\
\hline G3E3 & 15.00 & $15.63 \%$ & $69.83 \%$ \\
\hline G3E4 & 15.67 & $15.94 \%$ & $68.18 \%$ \\
\hline All treatments vs control & $* *$ & NS & NS \\
\hline Absolute control sample & $13.00 \mathrm{a}$ & $14.96 \%$ & $71.92 \%$ \\
\hline Grading & $14.81 \mathrm{~b}$ & $15.45 \%$ & $70.21 \%$ \\
\hline Elastomer & $14.81 \mathrm{~b}$ & $15.59 \%$ & $70.23 \%$ \\
\hline Coefficient of variation & 4.42 & 2.57 & 3.04 \\
\hline $\mathbf{P}$ & 0.0001 & 0.041 & 0.038 \\
\hline
\end{tabular}

$\left({ }^{*}\right)$ Significant difference between treatments.

$\left.{ }^{* *}\right)$ Highly significant difference between treatments. Common letters are not statistically different.

Source: Prepared by the author.

obtained $15.40 \%$ and $15.12 \%$ respectively. Low values of "\% voids in mineral aggregates" were obtained as well. The requirements set by the Ministry of Transport and Public Works of Ecuador (2002) do not establish maximum values to consider, therefore, it is wrong to say that the greater the voids in mineral aggregates (VMA), the more space available for asphalt layers. Thus, according to Carrasco (2004):

Existen valores mínimos para VMA [o VAM] los cuales están recomendados $y$ especificados como función del tamaño del agregado. Estos valores se basan en el hecho de que cuanta más gruesa sea la película de asfalto que cubre las partículas de agregado, más durable será la mezcla
[There are minimum values for VMA which are recommended and specified according to aggregate size. These values are based on the fact that the thicker the asphalt layer covering the aggregate particles, the more durable the mixture will be] (pp. 62-63).

6. Voids filled with asphalt. The average values of variable "\% voids filled with asphalt" are shown in Table 9. No statistical differences were determined for factor $E$, interaction GxE nor for contrast all treatments vs. control using the analysis of variance (ANOVA); however, statistical differences were determined for factor $\mathrm{G}$. Significance test conducted on factor $\mathrm{G}$ determined two statistical similarity features, where G3 stood out with $68.47 \%$, followed by G2 with $70.36 \%$ and, lastly, G1 with $71.80 \%$. Thus, 
results comply with the requirements of the Marshall Method, which establishes a minimum value of $65 \%$ and a maximum of $75 \%$ (Ministerio de Transporte y Obras Públicas del Ecuador, 2002).

\section{CONCLUSIONS}

1. The addition of $4 \%, 8 \%$ and $12 \%$ of elastomer to the design of asphalt mixtures with aged asphalt pavement resulted in a positive outcome, which make them suitable for field use in modified asphalt mixtures.

2. Average and fine grading, in the design of asphalt mixtures with aged asphalt pavement, helped achieve positive results regarding the reuse of elastomer and asphalt pavement, and therefore can be used in field use in modified asphalt mixtures.

\section{REFERENCES}

[1] Amorim, E. \& Lima, L. (2018). Uso de residuos de caucho en pavimentos de asfalto: una revisión de literatura. Revista científica multidisciplinaria base de conocimiento, 3(2), 39-47.

[2] Bertalot, E. (2017). Una mirada al reciclaje de neumáticos fuera de uso. Revista SLTCaucho, (19), 18-19.

[3] Bureau of International Recycling (BIR) (2016). Neumáticos. Retrieved from http://www.bir.org/ industry-es-es/tyres-es-es/

[4] Castro, V. (2015). Estudio de factibilidad de creación de una empresa recicladora de neumáticos desechados, para la producción de caucho modificador de asfalto, en la provincia de Guayas (Master Thesis). Universidad de Guayaquil, Ecuador.

[5] Centro de Estudios y Experimentación de Obras Públicas (CEDEX) (2011). Reciclado de pavimentos asfálticos. Madrid, Spain: Centro de Estudios y Experimentación de Obras Públicas.

[6] Figueroa, A., Fonseca, E., Amaya, C. \& Prieto, M. (2008). Contrastación entre el asfalto modificado con poliestireno y llanta triturada empleando dos métodos de mezclado. Revista Épsilon, (10), 67-79.

[7] Garnica, P., Delgado, H., Gómez, J., Alonso, S. \& Alarcón, H. (2004). Aspectos del diseño volumétrico de mezclas asfálticas. Sanfandila,
Mexico: Secretaría de Comunicaciones y Transportes / Instituto Mexicano del Transporte.

[8] Grytsenko, A., Pozdniakova, Y. y Vnukova, N. (2015). Estimation of possibility of tyre utilization products usage as alternative fuel. Recuperado de https://dspace.khadi.kharkov.ua/dspace/ bitstream/123456789/1082/1/\%D0\%90\%D0\% A2_36_07.pdf

[9] Lacamara, M. (2014). Brasil estrena el asfalto ecológico. Twenergy. Retrieved from https:// twenergy.com/a/brasil-estrena-el-asfaltoecológico

[10] Lecitra, M. (2010). Reducir, reutilizar y reciclar: el problema de los residuos sólidos urbanos. Retrieved from https://geic.files.wordpress. com/2010/10/reducir-reutilizar-y-reciclar.pdf

[11] Martín, A. (2015). Aplicación del caucho reciclado como solución constructiva ecológica. Recuperado de https://riunet.upv.es/bitstream/ handle/10251/55735/MART\%C3\%8DN\%20 -\%20Aplicaci\%C3\%B3n\%20del\%20caucho\%20 reciclado $\% 20$ como $\% 20$ soluci $\%$ C3\%B3n $\% 20$ constructiva $\% 20$ e c ol \% C $3 \%$ B 3 g i ca . pdf? sequence $=1$

[12] Ministerio de Transporte y Obras Públicas (MTOP) (2002). Especificaciones generales para la construcción de caminos y puentes. Quito, Ecuador: Ministerio de Obras Públicas / República del Ecuador.

[13] Paiva, G \& Ramos, G. (2014). Reciclado de pavimentos asfálticos y su reutilización para el diseño de mezcla de asfalto en caliente (Bachelor Thesis). Universidad Señor de Sipán, Peru.

[14] Peláez, G., Velásquez, S. \& Giraldo, D. (2017). Aplicaciones de caucho reciclado: una revisión de la literatura. Revista Ciencia e Ingeniería Neogranadina, 27(2), 27-50.

[15] Sánchez, R. (2012). Segunda vida de los neumáticos usados. Revista Química Viva, 11(1), 25-40.

[16] Carrasco, D. (2004). Estudio comparativo entre mezclas asfálticas en caliente y mezclas asfálticas con emulsiones tibias. (Bachelor Thesis). Universidad de Piura, Peru. 